\title{
Comunicación y patrimonio cultural. Comunicación patrimonial en instituciones museísticas de Río Gallegos
}

\section{Communication and cultural heritage. Heritage communication in museum institution of Rio Gallegos}

\author{
Natalia Marcial, Cristian Bessone \\ natalia86@hotmail.com,cristianbessone@hotmail.com
}

Universidad Nacional de la Patagonia Austral - Unidad Académica Río Gallegos

Av. Piloto "Lero" Rivera y Av. Gdor. Gregores

Río Gallegos - Santa Cruz - Argentina

Recibido: 16/04/2020. Aceptado: 11/09/2020

\section{RESUMEN}

Tanto la puesta en valor como la gestión del patrimonio se desprenden del entramado de sentidos que surgen del cruce que se haga a partir de su conceptualización. La sociedad contemporánea integra cada vez más distintas tecnologías para administrar, transmitir y almacenar información, cultura y, en gran medida, conocimiento. Por eso, el entorno social y cultural requiere que los museos adopten nuevos modelos de gestión. Estas nuevas formas de administración incluyen la necesidad de que los museos identifiquen y se comuniquen eficazmente con los diversos públicos que consideran importantes para el desarrollo de su actividad.

Siguiendo este enfoque, el presente trabajo expone los resultados de una investigación que analiza la situación de comunicación en las instituciones museísticas de la ciudad de Río Gallegos (Argentina). En el mismo, se indaga acerca de cómo se producen las diferentes funciones de comunicación, tanto en su estructura como en sus herramientas o medios más utilizados, al mismo tiempo que se evalúa su planificación y evaluación.

La aplicación en este caso de una metodología que combina encuesta con entrevista, permite pensar en la importancia de crear las condiciones para que los posibles usuarios entren en contacto con el bien o el servicio cultural deseado (Valdés, 1999)

Palabras Clave: patrimonio; gestión del patrimonio; museos; comunicación del patrimonio.

\begin{abstract}
Both the enhancement and the management of heritage emerge from the network of meanings that arise from the intersection made from its conceptualization. Contemporary society increasingly integrates different technologies to manage, transmit and store information, culture and, to a large extent, knowledge.

For this reason, the social and cultural environment requires museums to adopt new management models. These new forms of administration include the need for museums to identify and communicate effectively with the diverse publics that they consider important for the development of their activity.

Following this approach, this work presents the results of an investigation that analyzes the communication situation in the museum institutions of the city of Río Gallegos (Argentina).
\end{abstract}


In it, it is investigated about how the different communication functions are produced, both in their structure and in their most used tools or means, at the same time that their planning and evaluation is evaluated.

The application in this case of a methodology that combines a survey with an interview, allows us to think about the importance of creating the conditions for potential users to come into contact with the desired cultural good or service (Valdés, 1999)

Keywords: heritage; wealth management; museums; heritage communication.

\section{INTRODUCCIÓN}

El patrimonio cultural ha despertado grandes controversias, especialmente a partir de las últimas décadas del siglo XX. La conceptualización sobre aquello que es y no es patrimonio sigue dando lugar a numerosos debates. Sin embargo, la interculturalidad ha propuesto nuevas miradas sobre fenómenos pasados y presentes, sobre intercambio y enriquecimiento permanente a partir de la incorporación del otro, y de lo otro.

En la actualidad, las investigaciones que comprenden la comunicación global del patrimonio suelen incluir análisis, desde una perspectiva multidisciplinaria, de aspectos tales como la interpretación, la didáctica y los medios que aseguran la plena accesibilidad emocional e intelectual al patrimonio cultural sin menoscabar la solidez científica (Mateos Rusillo, 2008)

En Río Gallegos (Santa Cruz, Argentina) existen varias instituciones encargadas del resguardo patrimonial, como los museos, que son entidades centradas en la conservación, investigación (algunas de ellas), y difusión de los bienes culturales a los que se abocan, y tienen como una de sus funciones principales la comunicación con el entorno inmediato con el que se relacionan. La hipótesis que guía el trabajo, es que a pesar de la relevancia de esta última función y de su reconocimiento, en dichas instituciones no se ha logrado incorporar modelos de comunicación que sean capaces de asegurar el acceso al conocimiento y al propio patrimonio a una gran parte de la población.

Por esto, el presente trabajo ${ }^{1}$ se plantea como objetivo principal conocer y relevar la situación comunicativa en las instituciones museísticas del ámbito local, a fin de poder dar cuenta de aquellas problemáticas existentes en la estructura, planificación, y en las herramientas de comunicación más utilizadas.

El análisis de los mismos, permitirá reflexionar sobre los elementos que propician la plena democratización del acceso al patrimonio cultural. Además de comprender que debatir sobre estas problemáticas, constituye un primer paso para encontrar colaborativamente caminos que permitan generar conciencia de la importancia de la comunicación estratégica en las organizaciones culturales.

\section{ESTRUCTURA DEL DOCUMENTO}

El desarrollo de esta investigación se estructura de la siguiente manera: en la sección 2 se establece el marco teórico, donde se define los conceptos relacionados al patrimonio cultural y a los diferentes aspectos de su gestión. En la sección 3, se describe la muestra seleccionada

${ }^{1}$ El desarrollo de esta investigación se realiza por la autora en el marco del Proyecto de Investigación (PI) 29/A398 "Revitalización de los saberes populares tradicionales como patrimonio cultural inmaterial en la provincia de Santa Cruz", y se apoya en la Beca de Iniciación a la Investigación otorgada por la Universidad Nacional de la Patagonia Austral., Unidad Académica de Río Gallegos (UNPA-UARG) 
y su correspondiente instrumento de recolección de datos. Seguida de ella, en la sección 4, se exponen los resultados más significativos en base a los tres ejes temáticos que se plantearon para la cedula de investigación. En la sección 5, se realiza la discusión donde se expone la significación de los resultados que se han descripto. En la sección 6, se presenta la conclusión del trabajo que resume el contenido y el propósito de este estudio. Finalmente, en las secciones 7 y 8 se exponen anexos y bibliografía utilizada.

\section{MARCO TEÓRICO-CONCEPTUAL}

La palabra patrimonio, tan antigua como las grandes civilizaciones de la humanidad, hace referencia a "la propiedad de los bienes recibidos de nuestros antepasados" (Limón Delgado, 1999, p. 8). Esta concepción operativa del patrimonio, retoma la noción de construcción social, ya que lo que heredamos no es fijo, sino que se reconstruye en privado, en común y en el tiempo.

Para Prats (1997), el factor determinante que define al patrimonio tal cual lo entendemos hoy, es su significación simbólica, su capacidad para representar simbólicamente una identidad. Esto es lo que explica el cómo y porqué se movilizan recursos para conservarlos y exponerlos. La UNESCO (2014), reconoce como patrimonio cultural a los monumentos, los conjuntos arquitectónicos, los lugares (tanto obras del hombre, como obras conjuntas del hombre y la naturaleza) que tengan un valor universal excepcional desde el punto de vista de la historia, del arte o de la ciencia. Asimismo, este concepto se ha ampliado y clasificado en dos grandes grupos, por un lado, lo tangible (objetos, edificios) y, por lo tanto, material. Por el otro lado, lo simbólico, los saberes y creencias, que dota de sentido a los objetos, lo inmaterial.

El primero corresponde a la concepción originaria de patrimonio cultural, incorporada en la definición de la UNESCO, que involucra a todos los bienes culturales adheridos físicamente al territorio. Es decir, aquellos objetos que están de alguna manera relacionados con la historia, ya sea por ser representativos de algún proceso, por haber pertenecido a algún personaje trascendente o simplemente por su antigüedad. En esta última categoría suelen estar los objetos que encontramos exhibidos en los museos.

Por su parte, en la Convención para la Salvaguardia del Patrimonio Cultural Inmaterial de la UNESCO (2003), el patrimonio cultural inmaterial es definido como:

los usos, representaciones, expresiones, conocimientos y técnicas -junto con los instrumentos, objetos, artefactos y espacios culturales que les son inherentes- que las comunidades, los grupos y en algunos casos los individuos reconozcan como parte integrante de su patrimonio cultural

Sobre estas dos definiciones, gira todo un universo de conceptos que complementan y añaden matices, pero que sin embargo no alteran su significado. Cada objeto, edificio, escultura o monumento, es la materialización de una representación simbólica, de aquello que se despierta en nosotros al interactuar con esa materialidad.

Ahora bien, ¿cómo se protege los bienes culturales? Hablamos aquí de gestión del patrimonio o gestión patrimonial, que refiere a "acciones puntuales a través de un proceso de selección de situaciones o elementos, investigación, protección, puesta en valor y difusión" Kulemeyer y Estruch (2009, p. 20). De este modo, aunque existan grandes recursos si no están identificados, documentados, conservados, y si no se difunden y comunican, no cumplen con la función social por la que se definen.

Mateos Rusillo (2009), divide la gestión patrimonial en tres grandes áreas funcionales: la conservación, la investigación y la comunicación global, siguiendo éstas una secuencia unidireccional e inalterable. Así, la comunicación no solo forma parte del proceso de gestión 
del patrimonio, sino que juega un papel crucial al significar la imprescindible conclusión de cualquier intervención sobre los bienes patrimoniales.

A partir de esta perspectiva y dado el carácter comunicacional que guía el presente trabajo, se analiza con especial énfasis a la comunicación global del patrimonio. El concepto ha sido acuñado por Mateos Rusillo (2008, p. 14; 2009, pp. 27-29) al incluir bajo un mismo paraguas dos ideas fundamentales que, hasta entonces, habían sido tratadas por separado.

Por un lado, la difusión cultural que busca la plena accesibilidad intelectual y emocional del patrimonio mediante el uso de disciplinas como la interpretación y la didáctica, que incluye a su vez estrategias de difusión como la de difusión preventiva. Y, por otro lado, la ejecución de la comunicación, cuya finalidad sea atraer y fidelizar al público destinatario para que éste conozca y visite el recurso patrimonial.

En este contexto, la comunicación del patrimonio abarca un conjunto de formas diversas, que puede asumir la trasmisión y representación de bienes y obras culturales, que no se limita a la difusión de los mismos, sino que implica la creación de las condiciones para que los posibles usuarios entren en contacto con el bien o servicio cultural deseado, es decir, cumple con la función de aproximar el patrimonio cultural a la población (Valdés, 1999). No obstante, la mayoría de las instituciones patrimoniales no cuenta con modelos de comunicación que aseguren el conocimiento y el acceso a los bienes culturales para una gran parte de la población.

La apertura al gran público, o al menos a un abanico más amplio de personas que solo las previstas, es un continuo desafío porque, por un lado, representa una meta de democratización de los contenidos, pero que se ve condicionada por las diferentes barreras culturales (Mantecón, 2010). El desconocimiento de los museos por parte de una gran mayoría de los ciudadanos, es al mismo tiempo reforzado por una actitud negativa hacia ellas, dado que se suele construir una imagen de las mismas como entidades cerradas, elitistas y aburridas (Abugauch y Capriotti, 2012, p. 2).

Los museos, de acuerdo con el Consejo Internacional de Museos (ICOM), son: "instituciones permanentes, sin fines lucrativos, al servicio de la sociedad y de su desarrollo, abierta al público, que adquiere, conserva, investiga, comunica y expone el patrimonio material e inmaterial de la humanidad" (Art. $3^{\circ}$ Sección 1 del Estatuto del ICOM). Esta definición, elaborada en la 22a Asamblea General del año 2007, incorpora en su base al patrimonio inmaterial, ya que las dos facetas del patrimonio cultural no pueden ser gestionadas como aspectos separados: cada objeto expuesto en un museo, es la materialización de un matiz de la identidad de esa comunidad.

Los museos son, además, espacios de mediación y de comunicación pública que generan relatos orales, escritos, multimediales, interactivos, etc., en torno a y desde el museo, que transmiten una cosmovisión de épocas, tendencias y usos; responden a una visión particular (Martín-Serrano, 2008) de cómo es, fue o será el mundo, dentro de una muestra montada y exhibida.

Si analizamos esta afirmación, vemos como la comunicación toma mayor importancia considerándose un principio motor en la marcha del museo. Por este motivo, el entorno social y cultural actual requiere que los museos adopten nuevos modelos de gestión, nuevas formas de administración que incluyan la necesidad de que estos se identifiquen y se comuniquen eficazmente con los diversos públicos que consideran importantes para el desarrollo de su actividad. La comunicación participativa, la sindicación de contenidos, y ciertos espacios colaborativos podrían ser claves en el desarrollo de nuevas estrategias de comunicación cultural.

Para concluir, es en esta nueva era donde la comunicación del patrimonio deberá entenderse como una "acción integrada de comunicación" del recurso patrimonial (Capriotti, 2008, pp. 
134-135), que integre todos los mensajes y medios utilizados para trasmitir una identidad fuerte, clara y coherente del producto patrimonial.

\section{METODOLOGÍA}

En primer lugar, se debe aclarar que esta investigación tiene un marcado carácter cualitativo, con lo cual busca interpretar situaciones concretas acerca de la realidad social en la que se inserta nuestro objeto de estudio.

Aunque a priori, resulte accesible establecer la lista de los casos seleccionados para esta investigación, lo cierto es que no se dispone de un documento concreto en el que se contemplen todas las entidades museísticas, independientemente de su titularidad. Debido a ello, nuestra muestra consta de 13 instituciones conformadas por museos, monumentos históricos y el centro de interpretación, que fueron concebidos como espacios para la exhibición, promoción y conservación de los bienes patrimoniales. Los mismos, han sido relevados por la Escuela de Turismo de la Universidad de la Patagonia Austral- Unidad Académica de Río Gallegos (UNPA-UARG), y por la autora que lleva a cabo esta investigación.

A partir de esto, con el objetivo de obtener datos e información necesaria para realizar el estudio planteado, se realizó una cédula de encuesta general para todas las entidades donde se consideró tres grandes ejes temáticos: estructura, planificación y herramientas de comunicación. En forma complementaria, se realizaron entrevistas semiestructuradas a los representantes o encargados de cada institución.

En este sentido, cabe mencionar que a través de este método se buscó la comodidad del entrevistado para obtener no sólo las opiniones sobre los temas planteados, sino también información adicional.

\section{RESULTADOS}

De acuerdo a las respuestas obtenidas por las instituciones museísticas de Río Gallegos, los resultados están organizados en base a los tres ejes temáticos antes mencionados en la metodología. Pero antes de ello, y a modo de introducción exponemos una breve descripción de los 13 museos relevados. (Ver Cuadro 1)

\section{Cuadro 1}

\section{Institución/}

Museo

Principales actividades y horario de visita

\section{Museo de los Pioneros}

Es la casa más antigua de la ciudad. Permanece abierto todo el año, de lunes a domingo de 10 a 17 hs. Cuenta con una exposición permanente, además de muestras temporales, que son recorridas de forma guiada a turistas y a las escuelas del ámbito local. Es gestionado por la Municipalidad de Río Gallegos. 


\section{Museo Padre Jesús Molina}

Refleja el acervo cultural y natural de la provincia desde una perspectiva científica. Entre las actividades que se desarrollan son visitas guiadas, exposiciones temporales y permanentes tanto en el interior, como en el exterior, capacitaciones y proyectos institucionales. El horario de visita es de lunes a viernes de 9 a 19, mientras que sábados, domingos y feriados es de 12 a 19 hs. Es gestionado por la Secretaría de Cultura de la Provincia de Santa Cruz.

\section{Casa Histórica Gobernador Gregores}

Comprende parte del complejo habitacional de Santa Cruz que fue construido durante el gobierno del teniente Juan M. Gregores entre 1932-1945. Se realizan visitas guiadas en la vivienda, y en forma complementaria se ofrece al público el "circuito histórico de la gobernación", que consiste en un recorrido por la obra pública de su gestión. El horario de atención es de lunes a viernes de 10 a 17 hs. Es gestionado por la Provincia de Santa Cruz.

\section{Museo de Guerra Malvinas Argentinas}

Procura mantener vivo el recuerdo de la gesta de Malvinas en homenaje a quienes combatieron. Las visitas al museo son guiadas y consisten en la explicación de tres aspectos: geográfico, histórico y jurídico. Su apertura es de lunes a viernes de 11 a 16, en tanto sábados, domingos y feriados de 10 a 17 horas. Es gestionado por la Provincia de Santa Cruz.

\section{Museo Histórico Policial Crio. Gral. Roberto Godfrid}

Busca mantener el recuerdo y la memoria de la fuerza. El museo cuenta con una exhibición permanente y temporal, donde se realizan recorridos guiados para el público en general. El horario de visita es de lunes a viernes de 9:30 a 13 y de 14 a 20. Sábados de 10:30 a 13 horas. Es gestionado por la Policía de Santa Cruz.

\section{Museo Marítimo y Naval de la Patagonia Austral}

Forma parte del primer complejo habitacional de la ciudad, y procura proteger la historia de la Armada Argentina desde su traslado a Río Gallegos. Dispone de 3 salas pequeñas con muestras permanentes que son guiadas. Permanece abierto de lunes a viernes de 7 a 14 horas. Es gestionado por la Armada Argentina.

\section{Museo Prefectura Naval Argentina}

Es la primera Institución del Estado nacional que se instala en Río Gallegos. Toma su nombre y su fundación a partir del establecimiento de la Subprefectura. Cuenta con un solo ambiente, donde se erige una exhibición permanente que es recorrido de forma guiada. El horario programado es de lunes a viernes de 7 a 14 horas. Es gestionado por la Prefectura Naval Argentina. 


\section{Museo de Informática (UNPA-UARG)}

Trata de acercar la historia de la computación y la digitalización de la información hacia el interior de la comunidad universitaria, como hacia el exterior de la universidad. El museo mantiene una muestra permanente, y organiza visitas guiadas con una propuesta pedagógica a los distintos grupos escolares. La apertura se realiza por solicitud previa. Es gestionado por la Universidad Nacional de la Patagonia Austral, Unidad Académica Río Gallegos.

\section{Museo de Arte Eduardo Minnicelli}

Reúne y conserva el patrimonio artístico de la provincia, para desarrollar desde la niñez la sensibilidad hacia el arte y la cultura. En el mismo se realizan exposiciones temporarias (mensuales) y patrimoniales (una o dos veces al año). Además, cuenta con visitas guiadas, capacitaciones, talleres y proyectos institucionales. El horario de visita es de martes a viernes de 9 a 18, mientras que sábados y domingos es de 15 a 19 horas. Es gestionado por la Provincia de Santa Cruz.

\section{Museo Ferroviario Roberto Galián}

Busca valorar y preservar la vida del ramal ferro-industrial que funcionó en épocas de arduo trabajo minero. Cuenta con salas de muestras permanentes que son recorridas a través de una visita guiada. En el mismo se desarrollan otras actividades como la feria del libro independiente y autogestiva (FLIA). Puede visitarse de lunes a viernes de 14 a 17 horas. Es gestionado por la Asociación Amigos del Tren.

\section{Fundacruz, Casa de la Cultura}

Es un espacio destinado a las artes visuales y a las manifestaciones culturales de la ciudad de Río Gallegos. Dispone de nueve exposiciones anuales, dado que comienzan sus actividades de marzo a diciembre. Dentro de sus actividades se realizan talleres, charlas, servicios de té, etc. El horario de visita es viernes, sábados y domingos de 17 a 20 horas. Gestionado por la asociación no gubernamental Fundacruz.

\section{Centro de Interpretación Ambiental y Estuario de Río Gallegos (CIERG).}

Es un área concebida como un escenario para la comunicación, la motivación, la enseñanza y el aprendizaje sobre el patrimonio cultural y natural. La exhibición está compuesta por módulos interactivos con pantallas táctiles, rodillos, maquetas, libros, etc. La visita se realiza en forma guiada. El horario de atención es de lunes a sábados de 14 a 18 horas. Es gestionado por la Asociación Ambiente Sur. 


\section{Casa Histórica del Instituto María Auxiliadora (IMA)}

Es unas de las casas primitivas que data de 1901, y que fue declarada monumento histórico nacional en el 2008. Dispone de una planta alta y baja, donde a través de una visita guiada se recorre la arquitectura del lugar. El horario de visitas es lunes, miércoles y viernes de 13 a 17 horas. Es gestionado por el Instituto María Auxiliadora.

\subsection{Estructura}

En primera medida, se relevaron elementos que hacen a la estructura de funcionamiento de cada institución, y su relación con las actividades de comunicación. Para ello se identificó el tipo de administración o forma de gobierno que prevalece en cada museo de la muestra. En el siguiente gráfico se sistematiza los resultados:

Gráfico 1: Titularidad de los museos

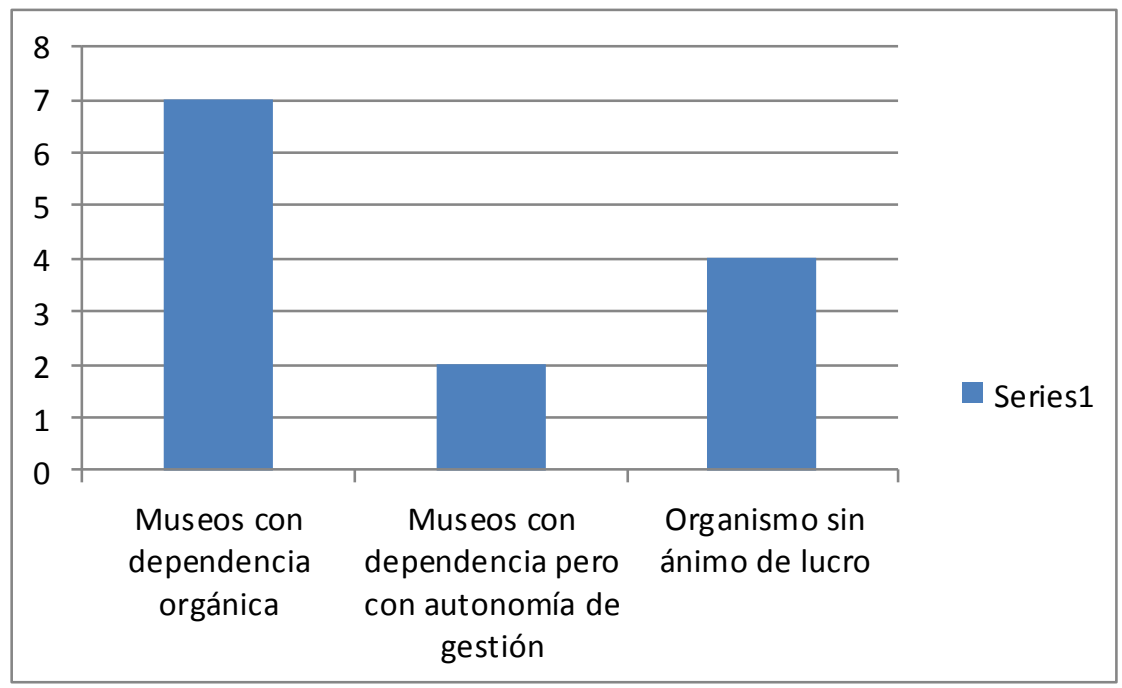

Fuente: elaboración propia

De las 13 instituciones, siete de ellas mantienen una dependencia orgánica con alguna institución de la administración pública. Es decir, forman parte indisociable de un departamento de la administración pública o tienen una relación estrecha con la misma. En este grupo, se encuentra el Museo de los Pioneros que responde a una gestión municipal, mientras que el Museo Padre Jesús Molina, la Casa Histórica Gobernador Gregores, el Museo de guerra Malvinas Argentinas, y el Museo Histórico Policial Gral. Roberto Godfrid, pertenecen a una entidad pública provincial. Asimismo, el Museo Marítimo y Naval de la Patagonia Austral y el Museo Prefectura Naval Argentina, depende de un organismo público nacional, en estos casos pertenecientes al Ministerio de Defensa de la Nación.

Por su naturaleza, este tipo de instituciones no gozan de una autonomía administrativa ni económica, por lo cual la estructura de gestión habitual posiciona a un director como responsable de las actividades del museo (cargo generalmente otorgado por la autoridad tutelar).

Por otro lado, la financiación proviene directa y casi exclusivamente del ente al que pertenecen. En este sentido, los resultados demuestran que se destinan recursos humanos, pero se confiere un bajo presupuesto al área de la comunicación. 
Siguiendo esta clasificación, identificamos dos casos particulares de instituciones con dependencia orgánica, pero con autonomía de gestión. Los mismos son: el Museo de Informática de la UNPA-UARG y el Museo de Arte Eduardo Minnicelli. El primero, depende de una entidad pública nacional, pero mantiene grandes líneas de actuación. Sus principales fuentes de subvención, provienen de proyectos y programas de extensión, por tal motivo no se destinan los recursos necesarios para la comunicación. Además, dicha institución no cuenta con personal propio, ni remunerado.

El segundo caso, el Museo Minnicelli corresponde al Consejo Provincial de Educación (C.P.E), y en su gestión combina planteamientos públicos con una metodología privada. Esta característica se manifiesta por una parte en los fondos que adquiere la institución que son tanto públicos como de particulares (sponsors). Y por otra, en el funcionamiento interno de la misma que es análogo al de una empresa privada. Sin embargo, a pesar de estas posibles ventajas en la administración, en los últimos años no obtuvieron aportes para el desarrollo de la comunicación, por ello en la actualidad se circunscribe exclusivamente a lo digital.

Por último, detectamos cuatro instituciones que son del tipo de organizaciones sin ánimo de lucro. Entre ellos, se encuentra el Museo Ferroviario Roberto Galián, conformado por la Asociación Amigos del Tren, las organizaciones no gubernamentales (ONG) Fundacruz, Casa de la Cultura y el Centro de Interpretación Ambiental y Estuario de Río Gallegos (CIERG). Así también, dentro de esta tipología el ICOM incluye a los museos de casas religiosas, como la Casa Histórica del Instituto María Auxiliadora (IMA), que si bien mantiene una relación directa con el establecimiento posee autonomía en su gestión. Para estas instituciones, la estructura comunicacional y administrativa depende de un responsable o encargado, que es designado generalmente por los miembros de la comisión organizativa. En tanto, la obtención de recursos procede del aporte de socios, particulares y de proyectos que tienen como finalidad la conservación y protección de los bienes que poseen.

A partir de estas características, actividades que desarrollan, planificación y apertura al público, clasificamos a las entidades museísticas como: de mayor actividad, mediano desarrollo y de desarrollo incipiente. (Ver Cuadro2)

\section{Cuadro 2.}

Instituciones de mayor actividad

Instituciones de mediano desarrollo

Instituciones de desarrollo incipiente

- Museo Minnicelli

- Museo Padre Jesús Molina

- Museo de los Pioneros

- CIERG

- Casa Gobernador Gregores

- Museos Malvinas Argentinas

- Museo de Informática

- Fundacruz

- IMA

- Museo Ferroviario

- Museo Policial

- Museo Marítimo y Naval

- Museo Prefectura Naval

Fuente: elaboración propia 


\subsection{Planificación}

Los resultados de la investigación indican que, de las 13 instituciones, solo cuatro de ellas realiza una planificación de la comunicación a corto plazo. Esta consiste básicamente en el proyecto anual de actividades, donde se definen acciones puntuales como la adaptación de contenidos, exposiciones, invitaciones, publicaciones, etcétera.

En algunos casos, como el Museo Minnicelli, el Museo de Informática y el CIERG, se mantienen reuniones semanales a fin de poder analizar y evaluar las acciones previstas en el calendario anual. Pese a ello, y tal como menciona Abugauch y Capriotti (2012), la mayoría de las entidades museísticas no posee una planificación fuerte y consolidada, por lo que las acciones de difusión son puntuales y concretas para cada actividad.

Asimismo, se puede observar que en las instituciones de mediano desarrollo y de desarrollo incipiente, la difusión se realiza de forma aislada y sobre "la marcha", dado que no cuentan con las herramientas necesarias de comunicación, o bien porque no disponen de personal propio para la atención del público.

En cuanto a la implementación de programas didácticos, 10 de las instituciones revelan que emplean algún mecanismo para involucrar a sus visitantes en el espacio en el cual se insertan. Estos resultados, demuestran por un lado los cambios que se han dado en la concepción tradicional del museo, y por otra en la necesidad de atraer al público que los visita.

Ahora bien, en relación a este tema los datos obtenidos arrojan que el público que asiste a los museos, procede en su mayoría de los centros educativos, ya que responden al interés y a las necesidades propias de la institución. Esto se da, porque en los lineamientos provinciales de educación incorporan durante el tercer y el cuarto año los contenidos básicos en ciencias sociales y naturales. De manera tal, que la visita al museo amplía el horizonte de la enseñanza formal, al ofrecerles medios para aprender, distraerse y discutir.

En este punto, es importante señalar que no existe una correspondencia entre el público meta al que dirigen sus acciones y quienes los visitan realmente. De los 13 casos analizados, 11 de ellos manifestaron dirigirse hacia el público en general, pero no diseñan habitualmente estrategias de comunicación que intenten captar esos nichos del mercado. Esto hace presuponer, que varias de estas instituciones no tienen definido el público objetivo, por lo que sus visitantes suelen ser ocasionales, no entienden el código del museo y solo buscan una interacción social y de entretenimiento.

Por otro lado, se puede observar que todas las entidades museísticas poseen cuadernos de visita, pero solo las de mayor actividad y mediano desarrollo utilizan a los mismos como un medio para conocer la proyección del museo en el tiempo.

Respecto de qué estrategias emplean para acercar a esos públicos, ilustramos algunas respuestas que mantienen correspondencia con otras entidades museísticas. (Ver Cuadro 3)

\section{Cuadro 3.}

¿Qué estrategias se emplean para acercar a esos públicos?

- Invitaciones del programa de actividades que se realiza en la institución a la dirección de los establecimientos educativos, a través de mails y llamados telefónicos.

- Recomendación directa a profesores que conocen la institución y participan de algunas de sus actividades.

- Adaptación de contenidos para los diferentes niveles escolares, como para personas con movilidad reducida o disminución visual.

- Proyectos educativos, como "el museo va a la escuela" del Museo Minnicelli, COM.UNI.D.A.D del CIERG, entre otros.

- Difusión a través de redes sociales, como también en medios televisivos y gráficos 
Las respuestas arriba desarrolladas apuntan algunas consideraciones que se hace necesario analizar, por ejemplo: la recomendación personal, para estas instituciones es de gran efectividad, dado que reduce la incertidumbre que tienen las personas antes de visitarlos. Además, para los museos que cuentan con escasos recursos de comunicación, el "boca a boca" es un recurso muy valioso que permite superar esas limitaciones.

Así también, la adaptación de los contenidos para los distintos niveles de público, hace pensar en una de las principales funciones de los museos, que es la investigación, aunque en la práctica solo las entidades de mayor actividad pueden desarrollar en parte este aspecto.

En tanto, acerca de qué público resulta difícil de interpelar, los resultados muestran que en general es un público joven adulto, de un rango etario de entre los 30 y 45 años de edad. A su vez, las personas con dificultades motoras o deficiencia visual, también encuentran limitaciones ante la falta de accesibilidad en el edificio y el recorrido de las salas.

\subsection{Herramientas de comunicación}

A nivel de la comunicación interna entre los miembros de las instituciones museísticas, se observa que 10 de las 13 entidades mantiene una interacción personal a través de reuniones informativas grupales, que se dan de forma semanal o mensual. Asimismo, estos casos manifiestan emplear nuevas formas de comunicación informal, como los grupos de trabajo por medio de la aplicación de Whatsapp.

Con respecto a las herramientas de comunicación más habituales, los resultados obtenidos indican que, en elementos tangibles, los folletos son lo más utilizados por las instituciones de mediano y menor desarrollo. Mientras que, seis de las 13 entidades afirmaron contar con flyers y gacetillas de prensa.

Por otra parte, en relación al manejo de las redes sociales que es uno de los principales vehículos de comunicación social, Facebook muestra un mayor uso por parte de las entidades museísticas. Seguida de ella, se sitúa Instagram que ha experimentado un mayor crecimiento durante el último año, especialmente en las instituciones de mayor actividad y de mediano desarrollo. Esto se debe, a que algunas de estas instituciones cuentan con personal a cargo de la difusión y a las posibilidades que le brindan estos medios en cuanto a información, ubicación, horarios, formas de contacto, servicios que ofrecen, catálogos de la colección o exhibición, etcétera.

A pesar de ello, la presencia que tiene la Web 2.0 en las entidades de menor desarrollo, es limitado porque solo funciona como una herramienta más de promoción, cuando en realidad debería redirigir los visitantes virtuales al museo físico.

En cuanto, a la forma de cómo se establece contacto con los medios de comunicación, todas las entidades expresaron que es directa con la institución, sobre todo en la inauguración de una muestra y en actividades específicas. Si bien, para los museos de menor actividad no es fluido, resulta de gran importancia por la cobertura masiva y la gratuidad del servicio. En relación a este tema, detectamos que solo tres de las instituciones, realiza publicidades pagas en medios televisivos o gráficos.

Otro elemento importante que señala esta investigación y que cobra relevancia a la hora de atraer a los visitantes, es la presencia de la identidad corporativa. Sobre este punto, podemos ver que seis de las 13 instituciones ha tenido una actualización en la identidad visual en los últimos diez años. Esto revela, por un lado, la importancia de captar la atención del público, y por otro la necesidad de diferenciarse de los demás a través de un logotipo adecuado que los distinga e identifique.

A su vez, dentro de esta gran área que hace a la comunicación externa, observamos que en ocho de las 13 entidades se dispone de señalización en la vía pública. La misma está centrada en referencias a la institución, dispuestos en la entrada a modo orientativo o en un punto estratégico de la ciudad, como ubicó el Museo Malvinas Argentinas. Sin embargo, estas 
herramientas son poco utilizadas por los centros museísticos, ya que constantemente son vandalizados.

Por último, en referencia a la pregunta qué cosas les gustaría continuar o mejorar, percibimos que para algunas instituciones se priorizó las necesidades institucionales por sobre las acciones a continuar o mejorar. A continuación, citamos algunos fragmentos de estas respuestas.

$>$ "Nos gustaría poder contar con una oficina técnica para todo el personal de la institución, y poner más en valor la casa." (Museo de los Pioneros)

$>$ "Contar con la presencia del municipio para disponer de personal propio para la atención del público" (Museo Ferroviario)

$>$ "Poder ampliar y cerrar la plazoleta, para que el museo tenga independencia de los vecinos" (Museo Prefectura Naval)

$>$ "Realizar más imitaciones de los barcos que tiene el museo, para que cuando salga a las exhibiciones no se deteriore" (Museo Marítimo y Naval)

$>$ "Poder refaccionar los elementos que están deteriorados dentro de la casa" (Casa Gregores)

$>$ "Queremos incorporar más dispositivos tecnológicos y mejorar la accesibilidad al museo" (Museo Malvinas Argentinas)

$>$ "Nos gustaría contar con más capacitaciones a través de las redes e incorporar más voluntarios" (CIERG)

$>$ "Disponer de más recursos humanos y diseñar una nueva imagen que sea independiente del colegio" (IMA)

$>$ "Poder implementar más dispositivos sonoros y visuales para mejorar de esta forma la accesibilidad al museo" (Museo Padre Molina)

$>$ "Queremos tratar de que las personas pierdan ese hermetismo que tienen con las fuerzas de seguridad para que nos visiten más" (Museo Policial)

$>$ "Seguir contando con el apoyo de los amigos de la casa y que se sumen más artistas del medio" (Fundacruz).

$>$ "Poder contar más personal para que el museo este abierto todos los días y mejorar los programas de accesibilidad para las personas con movilidad reducida o deficiencia visual" (Museo de Informática de la UNPA-UARG)

> "Nos gustaría en el área de la comunicación volver a tener la gráfica impresa, y por otro lado que tengan una mayor participación los sponsors" (Museo Minnicelli)

\section{DISCUSIÓN}

A partir de los resultados obtenidos, hacemos una revisión crítica y contextualizada. En primer lugar, siguiendo lo que describimos en el eje I acerca de la estructura, constatamos que en general las instituciones son pequeñas, en donde pocas personas realizan múltiples tareas y cumplen varios roles. En este sentido, la actividad de comunicación no suele diferenciarse netamente de otras actividades, y no siempre se la ubica en un lugar de relevancia. Esto, claramente no contribuye a la profesionalización del área y tampoco a la consolidación de la comunicación como una actividad estratégica de la que pueda tomarse un provecho intenso en estas instituciones.

También, observamos que hay una carencia casi generalizada en la planificación de la comunicación, solo los museos de mayor actividad disponen de un plan a corto plazo. Esto se debe, por una parte, a que los miembros de las instituciones desconocen cómo hacerlo, ya que mayormente quienes están a cargo de la difusión no cuentan con un perfil profesional de un 
comunicador. Por este motivo, no se ha logrado abarcar integralmente la comunicación a los modelos de gestión y valorización.

En este punto, es necesario señalar que un plan estratégico de comunicación apunta a la posibilidad de diseñar, gestionar y medir las acciones de comunicación posibles para esa organización en tanto sean pertinentes, dirigido a los públicos principales y que respondan a un presupuesto previamente establecido. Sin duda, estas recetas genéricas funcionan como una guía a seguir, pero en la práctica la mayoría de las instituciones realiza una planificación de la comunicación "sobre la marcha". Es decir, que suele pensarse en términos de acciones concretas para difundir una nueva exposición o actividad, y en el día a día cotidiano.

Por otra parte, la falta de financiamiento es uno de los principales problemas en estas entidades. Por ejemplo, para los museos con dependencia orgánica los cambios producidos en las gestiones de gobierno influyen directamente sobre la administración y la viabilidad económica. Del mismo modo, ocurre en las entidades con autonomía de gestión y sin ánimo de lucro donde las fuentes de subvención constituyen uno de los grandes desafíos. Cabe preguntarnos entonces algunos interrogantes para continuar indagando: ¿de qué forma puede un museo cumplir sus funciones sin recursos económicos y/o humanos?, ¿es necesaria la intervención del Estado en la gestión del patrimonio? Si no fuera la participación estatal directa ¿qué otras formas de organización podrían implementarse?

Otro elemento importante que detectamos en la planificación de la comunicación, es la falta de coherencia que se da entre el público hacia quienes dirigen sus acciones, y quienes lo visitan realmente. Sobre este tema, Davies (1996) apunta a tener una visión clara, determinar hacia dónde se dirige el museo y saber cómo llegar hasta allí. Esto asegura de alguna manera, que las entidades museísticas tengan el control de su destino, en lugar de limitarse a reaccionar sobre lo inmediato.

En referencia al último eje, destacamos el papel creciente de Internet y las redes sociales en las relaciones de los museos con sus públicos. Si bien permite mejorar la comunicación, observamos que en las entidades que no poseen estructuras de comunicación profesionalizadas solo se utiliza como un instrumento más de promoción. Pese a ello, se le supone un gran potencial en cuanto a facilitar relaciones más interactivas y colaborativas.

A nivel de la percepción por parte de los públicos, observamos que aún no podamos hablar de un modelo de comunicación con la sociedad que sea capaz de asegurar el conocimiento y el acceso del patrimonio museístico a gran parte de la población. Además, debemos añadir una doble barrera cultural que dificulta el acercamiento de la mayoría de los ciudadanos a los museos: por un lado, el desconocimiento mayoritario hacia estas entidades patrimoniales y, por otro, la actitud negativa predominante hacia ellas, fundamentada en la imagen que los museos tienen como lugares cerrados, elitistas y aburridos (Abugauch y Capriotti, 2012, p. 2) Finalmente, consideramos que esta investigación no queda acabada ni definitiva, ya que podría ser más valiosa si se completa con el análisis del comportamiento de los visitantes actuales, como de los potenciales. Por tanto, dejamos abierto el debate sobre todos los temas que se trataron y se desprenden de dicha investigación.

\section{CONCLUSIONES}

A modo de conclusión global, podemos decir que el haber indagado acerca de la estructura, planificación y herramientas de comunicación en las diferentes entidades museísticas, nos ha permitido no solo conocer sino también contribuir a este campo de conocimiento que hace a la comunicación del patrimonio cultural.

A partir del análisis de los resultados, constatamos que para la mayoría de las instituciones, la comunicación no ocupa un papel relevante dado que carece de modelos de gestión que 
posibiliten su implementación de manera fuerte, y a que comúnmente no suele diferenciarse a esta área como una actividad específica.

En este aspecto, la escasez de recursos económicos y la falta de apoyo del Estado ha sido señalado como una de las principales causas. Asimismo, no contar con profesionales especializados en el tema que proporcionen una visión estratégica, ha generado una insuficiencia en la planificación, como en la incorporación de planes o programas de medio y largo plazo.

Por esto, la mayor parte de los resultados de esta investigación indican que la comunicación es utilizada solamente como un instrumento de difusión de información, y no como una herramienta para relacionarse e interactuar con sus públicos.

Por otro lado, observamos que la comunicación digital de los museos en redes sociales, es vista principalmente como una oportunidad de maximizar el alcance de las acciones, y a la vez obtener un ahorro en los costos de comunicación. Esto implica, tratar de llegar a la mayor cantidad de personas promocionando la institución, pero no significa una posibilidad de crear las condiciones necesarias para que los posibles visitantes entren en contacto con el bien o servicio cultural deseado.

A pesar de ello, se han podido percibir varias iniciativas que apuntan a la accesibilidad de los museos, mediante la incorporación de rampas, información adaptada para personas con discapacidad, carteles con sistema Braile y audioguías. Razón por la cual, nos hace pensar en la responsabilidad social a la que deben dirigirse los museos para lograr la integración de todos los públicos en los espacios patrimoniales.

Consideramos que contar con esta información es de suma importancia, ya que la comunicación juega un papel fundamental en la democratización cultural de los museos, contribuyendo no solo a impulsar el conocimiento de los mismos, sino también a mejorar la interacción de estas entidades con sus públicos.

\section{REFERENCIAS}

ABUGAUCH E., y CAPRIOTTI P. (2012). Comunicación y Patrimonio Cultural. La gestión de la Comunicación en los museos de Argentina. Buenos Aires.

AGUDO TORRICO J. (1999). Patrimonio etnológico e inventarios. Inventarios para conocer, inventarios para intervenir. En E. Aguilar Criado (Ed.), Patrimonio Etnológico, Nuevas perspectivas de estudio. Granada: Instituto Andaluz del Patrimonio Histórico. Consejería de Cultua, Junta de Andalucía.

ÁLVAREZ P., BESSONE C., y KULEMEYER J. (2014). El nuestro social. Patrimonio y gestión. Apuntes de un proceso cambiante. Río Gallegos: UNPAEdita.

CAPRIOTTI P. (2008). La planificación estratégica de la comunicación del patrimonio cultural. In S. M. Mateos Rusillo (Ed.), La comunicación global del patrimonio cultural (pp. 133-154). Gijón (España): Trea.

INSTITUTO ANDALUZ DEL PATRIMONIO HISTÓRICO. (2008). Patrimonio cultural y medios de comunicación. Sevilla: Consejería de Cultura.

ICOM (2007). Estatutos del ICOM. Viena: ICOM.

KULEMEYER J., y ESTRUCH D. (2009). La gestión del patrimonio o el maravilloso arte de lo posible. Hermeneutic, 8. Recuperado de http://publicaciones.unpa.edu.ar/ index.php/1/article/view/10

LIMÓN DELGADO A. (1999). Patrimonio ¿de quién? En E. Aguilar Criado (Ed.), Patrimonio Etnológico, Nuevas perspectivas de estudio (pp 8-15). Granada: Consejería de Cultura. Junta de Andalucía. 
MANTECÓN A. R. (2010). Consumos culturales y ciudadanía en tiempos de globalización. En Indicadores Culturales 2009 - Cuadernos de Políticas Culturales. (pp. 90-99). Caseros, Argentina: Editorial de la Universidad Nacional de Tres de Febrero.

MARTÍN-SERRANO M. (2008). La mediación social. Madrid, España: Akal.

MATEOS RUSILLO S. M. (2008). Hacia una comunicación global del patrimonio cultural, o cómo potenciar su uso fomentando su preservación. In S. M. Mateos Rusillo (Ed.), La comunicación global del patrimonio cultural (pp. 19-50). Gijón (España): Trea.

MATEOS RUSILLO S. M. (2009b). El procés de comunicació global del patrimoni cultural. In S. M. Mateos Rusillo (Ed.), Comunicació del patrimoni cultural (pp. 5-36). Barcelona: UOC.

MATEOS RUSILLO S. M. (2009b). El procés de comunicació global del patrimoni cultural. In S. M. Mateos Rusillo (Ed.), Comunicació del patrimoni cultural (pp. 5-36). Barcelona: UOC. Mateos Rusillo, S. M. (2009c). La comunicació: La visualització del productes patrimonials. In S. M. Mateos Rusillo (Ed.), Comunicació del patrimoni cultural (pp. 5-35). Barcelona: UOC.

MATEOS RUSILLO S. M. (2013). Museos y Content Marketing. Hacia un nuevo modelo de generación de contenidos culturales. Revista Zer 34(18).

MONCLÚS GARRIGA C. (2005). La intervención en cultura. Principios que debe regir las políticas públicas. Primer Congreso Internacional sobre la Formación de los Gestores y Técnicos de Cultura. Valencia.

PRATS L. (1997). Antropología y patrimonio. Barcelona: Ariel.

RODRIGO M. (2008). Medios de comunicación e interculturalidad. Patrimonio cultural y medios de comunicación (pp. 180-189). Sevilla: Consejería de Cultura.

UNESCO (2003). Convención para la Salvaguardia del Patrimonio Cultural Inmaterial. Vienna: UNESCO.

UNESCO (2014). Indicadores de cultura para el desarrollo. Manual Metodológico. París: UNESCO.

VALDÉS M. (1999). La difusión cultural en el museo: servicios destinados al gran público. Gijón: Trea. 


\section{ANEXOS}

Comunicación y Patrimonio Cultural. Comunicación en instituciones museísticas de Río Gallegos

Institución:

\section{ENCUESTA:}

¿Qué actividades se desarrollan en el museo?

1. Estructura de la Comunicación

1.a ¿De quién depende el manejo de la comunicación?

$\square$ Responsable del Área

$\square$ Depende del director de la Institución

$\square$ Otro

1.b i Se destinan recursos (económicos, humanos) para la comunicación?

$\square$ Sí

No

2. Planificación de la comunicación en los Museos

2.a ¿Se planifica la comunicación?

$\square$ Sí

No

2.b ¿Cómo lo hacen?

$\square$ Realizan un conjunto de acciones coordinadas por todas las actividades de difusión

$\square$ Realizan acciones de difusión puntuales y concretas para todas las actividades y exposiciones.

Realizan acciones de difusión aisladas, solo para algunas actividades y exposiciones.

Otros

2.c ¿Quiénes participan de esas actividades?

2.d ¿Sobre qué cosas se planifica?

$\square$ Campañas de difusión de la colección permanente de la Institución

$\square$ Acciones de difusión de las exposiciones temporales de la Institución

Campañas de difusión de otras actividades de la Institución (cursos, etc.)

Campaña de difusión de la Institución a nivel general

Cuando se realiza una exposición, ¿se piensa en algún mecanismo para que el público pueda intervenir de alguna manera en el mismo?

2.e ¿Existe algún programa didáctico de actividades?
$\square$ Sí
No

2.f ¿Qué público los visita principalmente?

$\square$ Público en general de la ciudad

Centros educativos

Turistas

Otros museos o centros de exposiciones

Medios de comunicación

Otros 
2.g ¿Hacia qué públicos focalizan sus acciones?

$\square$ Público en general de la ciudad

$\square$ Centros educativos

Turistas

Otros museos o centros de exposiciones

Medios de comunicación

Otros

2.h ¿Qué estrategias emplean para acercar a esos públicos?

2.i ¿Qué público resulta difícil de interpelar? ¿Cuáles son las estrategias para acercarlos?

2.j i Quiénes le gustaría que los visitara?

¿Tienen cuadernos de visitas?

Sí

No

2.I ¿Qué se hace con ellos? Según su observación y comentarios de los visitantes ¿cree que los visitantes entienden lo que se expone? ¿por qué?

3. Herramientas de la comunicación

3.a ¿Cómo es la comunicación interna entre los miembros/voluntarios de la Institución?

$\square$ Interpersonal

Reuniones informativas grupales

$\square$ Cartelera interna de noticias

$\square$ Correo electrónico

Intranet de la institución

Otros

3.b ¿Qué herramientas de comunicación usan?

Folletos

$\square$ Flyers

$\square$ Gacetillas de prensa

$\square$ Comunicados al C.P.E

$\square$ Página web

$\square$ E- institucional

$\square$ Blog institucional

$\square$ Perfiles en redes sociales (Facebook, Instagram. Tweeter

Canales en web de videos (Youtube)

$\square$ Cuentas en web de fotografías

Otros

3.c ¿Qué importancia le asignan a las redes sociales? 
3.d ¿Se realizan publicidades en medios gráficos, radiales o televisivos?

$\square$ Sí

No

3.e ¿A través de qué forma se establece contacto con los medios masivos de comunicación?

$\square$ Notas/comunicados de prensa

Reuniones de trabajo

Contacto directo con la institución

Otro

3.f ¿Qué tipo de información se envía a los boletines culturales o informativos?

$\square$ Información de la Institución

$\square$ Información de la colección permanente

$\square$ Información de exposiciones temporales

$\square$ Información de las actividades de extensión cultural

Otro

3.g ¿Se realizó alguna actualización en la identidad visual de la institución?

Sí

No

3.h ¿Qué cosas le gustarían continuar o mejorar?

3.i ¿Existen elementos de comunicación que el visitante se lleve luego de cada exposición? ¿Cuáles?

3.j Comunicación en la vía pública

Reparto de volantes

$\square$ Reparto de folletos

$\square$ Carteles/flyers

$\square$ Vallas publicitarias

$\square$ Mobiliario urbano

$\square$ Otros 